\title{
Mitochondrial Protein in the Nucleus
}

\author{
Bor Luen Tang \\ Department of Biochemistry, Yong Loo Lin School of Medicine, National University of Singapore, Singapore \\ Email: bchtbl@nus.edu.sg
}

Received 25 May 2015; accepted 16 June 2015; published 19 June 2015

Copyright (C) 2015 by author and Scientific Research Publishing Inc.

This work is licensed under the Creative Commons Attribution International License (CC BY). http://creativecommons.org/licenses/by/4.0/

(c) (i) Open Access

\begin{abstract}
Other than the respiratory chain components, most mitochondrial proteins are synthesized in the cytosol and imported into the mitochondria. Many mitochondrial proteins therefore have at least a transient cytosolic appearance, and several have a dual mitochondrial-cytosol functional localization. However, recent work has revealed several proteins, one of which is a large protein complex, with dual mitochondrial and nuclear localizations. The enzyme fumarase which catalyzes the reversible hydration/dehydration of fumarate to malate is part of the mitochondria matrix tricarboxylic acid (TCA) cycle. It could, however, be recruited from the cytosol to the nucleus in response to DNA damage, where it is important for DNA repair. The pyruvate dehydrogenase complex (PDC) generates acetyl-CoA from pyruvate, and is recently shown to translocate from the mitochondrial matrix into the nuclear under mitogenic and stress conditions to generate acetyl-CoA within the nucleus. The mitochondrial monooxygenase CLK-1/COQ7 responsible for the synthesis of ubiquinone is most recently found to have a nuclear isoform with an uncleaved amino terminus, where it affects transcriptional changes associated with mitochondrial reactive oxygen species (ROS) generation. In this review, we highlight these unusual cases of nuclear localization of classically mitochondrial proteins, and discuss their possible functions in the nucleus.
\end{abstract}

\section{Keywords}

Fumarase, Pyrivate Dehydrogenase Complex (PDC), CLK-1, COQ7, Mitochondria, Nucleus

\section{Introduction}

The mitochondrion is derived from a prokaryotic $\alpha$-proteobacteria symbiont which has lost a large part of its original genome to the host nucleus [1]. Of the thousand or so protein in the mitochondrial, only a handful, most prominently the respiratory chain components, are mitochondrial DNA (mtDNA) encoded, and are transcribed/ translated locally. Most mitochondrial proteins are synthesized in the cytosol by cytoplasmic ribosomes, and their mitochondrial import is facilitated by a number of mitochondrial translocase complexes [2]-[4] localized at the mitochondrial outer and inner membranes. A nascent polypeptide precursor destined for mitochondrial im- 
port is typically kept in a denatured form by chaperones, and first enters the inter membrane space through the translocase of the Outer Membrane (TOM) complex. Those harboring an N-terminal mitochondrial targeting signal (MTS), or a "presequence", are then inserted into the inner mitochondrial membrane by the translocase of the Inner Membrane 23 (TIM23) complex. Translocation into the matrix requires the presequence translocase-associated import motor (PAM) and a membrane potential across the IMM.

For a growing group of canonically mitochondria-targeted proteins, mitochondrial residence is however not exclusive. Other than the mitochondria, these proteins could also be found in the cytosol, or even other organelles, such as the endoplasmic reticulum, chloroplast, peroxisomes and the nucleus. These dual targeting or localization of proteins occurs via a variety of mechanisms, which has been extensively reviewed [5]. Many proteins could shuttle between the cytoplasm and the nucleus. This is probably due to the fact that molecules smaller than 25 - $30 \mathrm{kDa}$ in size could partition rather freely between the two compartments, as the pores of the nuclear envelope are rather large and flexible [6]. Selective import of nuclear targeted proteins harboring nuclear localization signals (NLS) are typically facilitated by the importin family of adaptors and a gradient of activated Ran GTPase [7] [8], while nuclear export requires a machinery that recognizes a nuclear export signal (NES). Uni- or bidirectional nuclear-cytoplasmic shuttling is a critical aspect of multiple cellular signaling processes, and defects or dysregulation of these often has notable pathophysiological consequences [9].

Of the dual-localized proteins, those that reside at both the mitochondria and the nucleus are of particular emerging interest [10]. A good number of predominantly nuclear proteins are now known to be localized to and function at the mitochondria. Prominent amongst these are those whose activities impinge on cell death and survival, as well as senescence and aging. The tumor suppressor p53 is primarily known as a nuclear transcription factor mediating senescence and apoptosis, but it also accumulates in the mitochondrial matrix in response to oxidative stress and triggers mitochondrial permeability transition and necrosis [11]. Estrogen receptors $\alpha$ and $\beta$ are nuclear hormone receptors but could also be found in the mitochondria [12] where these have anti-apoptotic functions [13]. The telomerase reverse transcriptase (TERT), which counteracts replicative telomeric shortening of chromosomes in the nucleus, is also known to be targeted to the mitochondria under oxidative stress [14] where it protects mtDNA from damage [15] and performs an extension of its role in countering senescence and aging [16]. FoxO3a, a member of the Forkhead subclass O (FoxO) transcription factors, is also known to localized to the mitochondria where it functions in the regulation of processes ranging from differentiation [17] to apoptosis [18]. Within the mitochondria, FoxO3a interacts with and is deacetylated by the mitochondrial sirtuin Sirt3 [19]. However, FoxO3a and other FoxO isoforms (or Daf-16 in C. elegans) are also components of an evolutionarily conserved lifespan regulation axis [20] [21] involving the nuclear sirtuin Sirt1 [22] [23], and the transcriptional coactivator peroxisome proliferator activated receptor $\gamma$-coactivator $1 \alpha$ (PGC- $1 \alpha$ ), both of which have been shown to also localized to the mitochondria [24].

On the other hand, several recent findings have indicated that a small number of classically mitochondrial localized proteins could be also found at the nucleus, where they perform specific functions that were either related or unrelated to their roles in the mitochondria. In the ensuing paragraphs, we shall focus on three such proteins that are evolutionarily conserved-fumarase, the pyruvate dehydrogenase complex (PDC) and the mitochondrial diiron containing monooxygenase Clk-1/COQ7.

\section{Fumarase-A TCA Cycle Enzyme Important for DNA Repair in the Nucleus}

Fumarase, or fumarate hydratase (catalyzing a reversible conversion of fumarate to malate) is a well-known TCA cycle enzyme residing and acting in the mitochondria matrix, but there is also a cytosolic fumarase isozyme [5] [25]. The function of the latter has remained elusive. To investigate what functions might the cytosolic fumarase have, Yogev and colleagues generated a yeast strain with the nuclear fum 1 gene deleted, but with a copy of the gene inserted into the mitochondria genome. The resultant fumarase is thus exclusively mitochondria localized, and the strain has a fully functional TCA cycle and appear outwardly normal [26]. The only significant phenotype is a 10 - 100 fold increased in sensitivity to DNA damage caused by ionizing radiation and the DNA synthesis inhibitor hydroxyurea. The strain is also particularly sensitive to an inducible expression of site-specific double-stranded-DNA endonuclease, which generates DNA double strand breaks (DSBs). This sensitivity is reversed with the co-expression of a cytosolic from of fumarase without the mitochondrial targeting sequence, but the enzymatic activity must be intact. Fumaric acid, but not diethyl-malate, complements the phenotype resulting from absence of cytosolic fumarase. The Authors showed that fumarase activity in wild type yeast cells and human HeLa cells are both induced by hydroxyurea exposure. Interestingly, both hydroxyurea 
and ionizing radiation induced the translocation of fumarase into the nucleus of HeLa cells. Silencing of fumarase expression increased the sensitivity of a panel of human cell lines to DNA damage, and the DSB damage response, as gauged by phosphorylation of histone H2AX, is apparently impaired.

These findings revealed a previously unrecognized function of fumarase in DNA DSB repair in the nucleus. They also provided a potential explanation for the human fumarate hydratase (FH) gene being a susceptibility locus for a known genetic predisposition to Hereditary Leiomyomatosis and Renal Cell Cancer (HLRCC) [27]. FH therefore appears to have tumor suppressor-like properties, but it is not particularly clear how it might act in this regard. Fumarate accumulates in cells with FH mutational inactivation, and fumarate is known to stabilize the angiogenic hypoxia-inducible factor (HIF) by acting as a competitive inhibitor of HIF prolyl hydroxylase [28]. While this hinted at a mechanism associated with tumor angiogenesis, it does not fully explain the tumor suppressor function of FH. The latter is better understood by the discovery that FH activity and product at the nucleus is required for DSB repair. It is yet unclear how fumarase activity and it's product is important for DNA damage repair, and future investigations shall shed light on this and other related uncertainties, such as whether fumarase also participate in mtDNA repair.

\section{The Pyruvate Dehydrogenase Complex (PDC) and Acetyl-CoA Production in the Nucleus}

The pyruvate dehydrogenase complex (PDC) [29] catalyzes multiple enzymatic steps to generate acetyl-CoA from glycolysis derived pyruvate [30], which then feeds into the TCA cycle in the mitochondrial matrix. The PDC has 3 enzyme activities (E1, E2 and E3), each comprising of multiple polypeptide subunits, and is one of the largest multiprotein complexes known. The mammalian PDC is about $8-10 \mathrm{MDa}$ in size, and to put this in perspective, it is significantly larger than the largest complex in the mitochondrial electron transport chain and ribosomes [29] [31]. In an intriguing finding reported last year, it was demonstrated that the PDC is also present in the nucleus [32]. The PDC components E1, E2 and E3BP were immune-localized in the nucleus by their respective antibodies, and the Authors showed that nuclear PDC is functional in generating acetyl-CoA from pyruvate. A particularly unusual and intriguing point pertaining to nuclear localized PDC is their biogenic origin. PDC components are not detected in the cytosol in any abundance, and therefore there isn't any evidence for a cytoplasmic-nuclear translocation. However, nuclear PDC levels are significantly elevated in cells upon mitogenic stimulation and chemical stresses, and intriguingly, in a manner that is concomitant with a reduction in mitochondrial PDC levels. The Authors thus proposed that the mitochondrial PDC could be translocated, likely as an intact complex, from the mitochondrial matrix to the nucleus [32].

The mechanism of PDC's mitochondrial-nuclear translocation was not addressed by the Authors and remains unclear, except that this is dependent on the chaperone heat shock protein of $70 \mathrm{kDa}$ (Hsp70), which facilitates nuclear import and could associate with both PDC E1 and E2. While the nuclear pore complex is large and has tremendous flexibility to allow the passage of very protein complexes such as the ribosomal subunits, there is currently no known mechanisms that could allow the huge PDC to negotiate the inner and outer mitochondrial membranes. Interestingly, PDC-E2 has been previously shown to be nuclear-localized in T lymphocytes, where it interacts with the signal transducer and activator of transcription 5 (STAT5) and modulates the latter's transcriptional activity [33] It is also possible that PDC could be assembled outside the mitochondrial matrix, and there are some evidence for this in some cancer cells [34].

What are the functions of nuclear PDC? Unlike mitochondrial PDC, the nuclear form is not subjected to pyruvate dehydrogenase kinase (PDK) phosphorylation and inhibition. The Authors showed that acetyl-CoA generated by PDC in the nucleus is apparently important for histone acetylation, and nuclear PDC is required for S phase entry and cell cycle progression [32]. Although there are other mechanisms for the generation of acetylCoA within the nucleus, such as by the nuclear form of ATP citrate lyase using citrate [35], nuclear PDC's ability to generate acetyl-CoA from pyruvate may become important in times when the citrate pool is depleted or shifted towards lipid synthesis. At the moment, however, the precise function of PDC in the nucleus and its role in supplying acetyl-CoA for histone acetylation awaits further verification.

\section{Mitochondrial CLK-1/COQ7 with a Nuclear Transcriptional Activity?}

The C. elegans Clock abnormal protein 1 (CLK-1) and its human orthologue Coenzyme Q biosynthesis protein 7 (COQ7) encodes a mitochondrial di-iron containing monooxygenase [36] that catalyzes hydroxylation of 5-de- 
methoxyubiquinone to 5-hydroxyubiquinone [37]. Other than ubiquinone biosynthesis, CLK-1/COQ7 is also prominent as an evolutionarily conserved determinant of lifespan [38] [39]. The polypeptide harbors a MTS, and its function in mitochondrial respiration reinforces the traditional view that it is exclusively a mitochondrial protein. In a very recent report, Monaghan and colleagues have however documented the presence of CLK-1/ COQ7 in the nucleus [40]. The Authors immune-localized COQ7 in the nucleus of HeLa cells and CLK-1-GFP to the nucleus in transgenic worms, and identified a nuclear targeting sequence (NTS) adjacent to the MTS for COQ7. In the mitochondria, this sequence would have been cleaved as the MTS is proteolytically processed, and the Authors indeed observed a slower migrating uncleaved form of COQ7 in cells. Interestingly, this human NTS sequence of COQ7 is not conserved in the worm CLK-1, and nuclear targeting of CLK-1 in C. elegans may use other determinants. A CLK-1 mutant with its MTS deleted nonetheless became predominantly nuclear, and failed to rescue the defect in ubiquinone synthesis in clk-1 null worms.

What is CLK-1/COQ7 doing in the nucleus? A strong hint of their possible nuclear function came from the observation that nuclear accumulation of both orthologues were stimulated by ROS, and attenuated by the anti-oxidant $\mathrm{N}$-acetyl cysteine. Basal and induced ROS levels were increased in clk-1 null worms and human cells lacking nuclear COQ7, and both models had a decreased level of glutaminase but increased levels of the WW domain containing oxidoreductase (WWOX), which could be reversed by expression of the nuclear-restricted form of CLK-1/COQ7. In addition, a range of redox responsive genes such as SOD2 (encoding the mitochondrial superoxide dismutase) and NRF2 (encoding nuclear factor erythroid 2-related factor 2, a key mediator of anti-oxidant response pathways) and their worm orthologues were also increased in a manner that could be suppressed by nuclear-restricted CLK-1/COQ7. This ROS responsive nuclear targeting of CLK-1/COQ7 suggests that the proteins may act to regulate ROS signaling and metabolism [40].

As noted above, CLK-1/COQ7 is a longevity gene, with its mutations resulting in a prolonged lifespan. Interestingly, nuclear CLK-1 also contributes to C. elegans longevity independently of mitochondrial CLK-1, as expression of the nuclear targeted form is able to suppress the longevity phenotype of clk-1 null worms. The lifespan extension effect of CLK-1/COQ7 defects has been linked to activation of a unique mitochondria-nucleus retrograde signaling process, the mitochondrial unfolded protein response $\left(\mathrm{UPR}^{\mathrm{mt}}\right)$ [41] [42]. Indeed, expression of nuclear CLK-1/COQ7 suppressed a subset of UPR ${ }^{\mathrm{mt}}$ genes that are elevated in clk-1 null worms. Exactly how does CLK-1/COQ7 modulate UPR ${ }^{\mathrm{mt}}$ gene expressions? The Authors offered some very tantalizing evidence to suggest that CLK-1/COQ7 may directly affect gene transcription. They found that COQ7 associates with the chromatin, and a number of promoter sites, including that of WWOX and TIMM22 (encoding the mitochondrial import inner membrane translocase subunit Tim22), are enriched in COQ7 chromatin precipitates in ChIP analyses. If confirmed by future analysis, this will open another dimension in the cellular network of lifespan determining genes and gene products.

\section{Mitochondrial Proteins in the Nucleus-How Did They Get There and What Do They Do?}

In the paragraphs above, prominent examples of a small group of mitochondrial proteins that are localized and appear to function in the nucleus are described. Many interesting questions arise from these observations, and some of these are discussed below.

How did the mitochondrial proteins go to the nucleus? While canonical mitochondrial targeting signals are present in all these proteins, nuclear targeting signals, albeit non-canonical, have been identified only for COQ7 [40]. The dual targeting mechanisms of fumarase have been elaborately discussed [25]. Fumarase goes to the mitochondria by translation coupled import and a fraction of these could become cytoplasmically localized if they are folded prematurely (reverse translocation). It is still unclear as to how cytosolic fumarase is subsequently targeted to the nucleus in response to DNA damage. On the other hand, the process of mitochondrial-nuclear translocation proposed for the very large PDC is at the moment completely lacking in a plausible mechanism, and much more work is needed to confirm this mitochondria-nuclear translocation mode and to gain some mechanistic insights to how it might occur [43].

At the nucleus, the canonical enzyme activity of both fumarase and PDC are apparently required for their apparent function. Fumarase's production of fumaric acid is important for DSB repair, and acetyl-CoA generated by PDC aids cell cycle progression and proliferation. On the other hand, whether the enzymatic activity of CLK-1/COQ7 is necessary for ROS signaling and metabolism in the nucleus has not been determined. In any case, CLK-1/COQ7 appears to be able to directly regulate gene expression through their association with the 
chromatin. Of course, this likely involves other transcription factors and complexes that remained to be identified.

Incidentally, the Activating Transcription Factor associated with Stress-1 (ATFS-1), a major transcription factor mediating UPR ${ }^{\mathrm{mt}}$ in C. elegans, has also dual mitochondria and nuclear localization and function. ATFS-1 has both an NLS and a mitochondrial MTS [44]. During mitochondrial ROS stress, mitochondrial import of ATFS-1 is reduced, and a fraction of the protein accumulates in the cytosol and translocate to the nucleus. ATFS- 1 could bind to the promoter of both ATFS- 1 bound directly to gene promoters in both the nuclear and mitochondrial genomes during UPR ${ }^{\mathrm{mt}}$, and tunes a balance expression of oxidative phosphorylation related genes [45]. There are other mitochondrial proteins with a nuclear presence, although their exact function in the nucleus is less clear. One example is Nfs1, a highly conserved mitochondrial cysteine desulfurase involved in iron-sulfur cluster assembly as a sulfur donor [46]. Nfs1 acts in the mitochondrial as well as the cytosol, where it provides S for molybdenum cofactor biosynthesis [47]. The nuclear role for Nfs1 is unknown, but the gene is essential in yeast, and an Nsf1p mutant with a disrupted NLS could not complement cell growth defects of chromosomal Nsf1 deletion [48].

It is highly likely that we will see more examples in the near future of mitochondrial proteins being nuclear localized and functional. Deciphering the regulatory dynamics of these proteins and their respective functions in the two compartments will likely broaden our understanding of key mitochondrial-nuclear signaling processes underlying cell survival and aging.

\section{Acknowledgements}

The author declares no conflict of interest.

\section{References}

[1] Gray, M.W. (2012) Mitochondrial Evolution. Cold Spring Harbor Perspectives in Biology, 4, a011403. http://dx.doi.org/10.1101/cshperspect.a011403

[2] Rehling, P., Brandner, K. and Pfanner, N. (2004) Mitochondrial Import and the Twin-Pore Translocase. Nature Reviews Molecular Cell Biology, 5, 519-530. http://dx.doi.org/10.1038/nrm1426

[3] Dudek, J., Rehling, P. and van der Laan, M. (2013) Mitochondrial Protein Import: Common Principles and Physiological Networks. Biochimica et Biophysica Acta (BBA)-Molecular Cell Research, 1833, 274-285. http://dx.doi.org/10.1016/j.bbamcr.2012.05.028

[4] Qiu, J., Wenz, L.-S., Zerbes, R.M., et al. (2013) Coupling of Mitochondrial Import and Export Translocases by Receptor-Mediated Supercomplex Formation. Cell, 154, 596-608. http://dx.doi.org/10.1016/j.cell.2013.06.033

[5] Yogev, O. and Pines, O. (2011) Dual Targeting of Mitochondrial Proteins: Mechanism, Regulation and Function. Biochimica et Biophysica Acta (BBA)-Biomembranes, 1808, 1012-1020. http://dx.doi.org/10.1016/j.bbamem.2010.07.004

[6] Terry, L.J. and Wente, S.R. (2009) Flexible Gates: Dynamic Topologies and Functions for FG Nucleoporins in Nucleocytoplasmic Transport. Eukaryotic Cell, 8, 1814-1827. http://dx.doi.org/10.1128/EC.00225-09

[7] Tran, E.J., King, M.C. and Corbett, A.H. (2014) Macromolecular Transport between the Nucleus and the Cytoplasm: Advances in Mechanism and Emerging Links to Disease. Biochimica et Biophysica Acta (BBA)-Molecular Cell Research, 1843, 2784-2795. http://dx.doi.org/10.1016/j.bbamcr.2014.08.003

[8] Cautain, B., Hill, R., de Pedro, N. and Link, W. (2015) Components and Regulation of Nuclear Transport Processes. FEBS Journal, 282, 445-462. http://dx.doi.org/10.1111/febs.13163

[9] Mor, A., White, M.A. and Fontoura, B.M.A. (2014) Nuclear Trafficking in Health and Disease. Current Opinion in Cell Biology, 28, 28-35. http://dx.doi.org/10.1016/j.ceb.2014.01.007

[10] Czypiorski, P., Altschmied, J., Rabanter, L.L., Goy, C., Jakob, S. and Haendeler, J. (2014) Outfielders Playing in the Infield: Functions of Aging-Associated "Nuclear" Proteins in the Mitochondria. Current Molecular Medicine, 14, 1247-1251. http://dx.doi.org/10.2174/1566524014666141202125935

[11] Vaseva, A.V., Marchenko, N.D., Ji, K., Tsirka, S.E., Holzmann, S. and Moll, U.M. (2012) p53 Opens the Mitochondrial Permeability Transition Pore to Trigger Necrosis. Cell, 149, 1536-1548. http://dx.doi.org/10.1016/j.cell.2012.05.014

[12] Yager, J.D. and Chen, J.Q. (2007) Mitochondrial Estrogen Receptors—New Insights into Specific Functions. Trends in Endocrinology and Metabolism, 18, 89-91. http://dx.doi.org/10.1016/j.tem.2007.02.006

[13] Liang, J., Xie, Q., Li, P., Zhong, X. and Chen, Y. (2015) Mitochondrial Estrogen Receptor $\beta$ Inhibits Cell Apoptosis 
via Interaction with Bad in a Ligand-Independent Manner. Molecular and Cellular Biochemistry, 401, 71-86. http://dx.doi.org/10.1007/s11010-014-2293-y

[14] Saretzki, G. (2009) Telomerase, Mitochondria and Oxidative Stress. Experimental Gerontology, 44, 485-492. http://dx.doi.org/10.1016/j.exger.2009.05.004

[15] Haendeler, J., Dröse, S., Büchner, N., Jakob, S., Altschmied, J., Goy, C., Spyridopoulos, I., Zeiher, A.M., Brandt, U. and Dimmeler, S. (2009) Mitochondrial Telomerase Reverse Transcriptase Binds to and Protects Mitochondrial DNA and Function from Damage. Arteriosclerosis, Thrombosis, and Vascular Biology, 29, 929-935. http://dx.doi.org/10.1161/ATVBAHA.109.185546

[16] Ale-Agha, N., Dyballa-Rukes, N., Jakob, S., Altschmied, J. and Haendeler, J. (2014) Cellular Functions of the DualTargeted Catalytic Subunit of Telomerase, Telomerase Reverse Transcriptase-Potential Role in Senescence and Aging. Experimental Gerontology, 56, 189-193. http://dx.doi.org/10.1016/j.exger.2014.02.011

[17] Meshkini, A. and Yazdanparast, R. (2012) Foxo3a Targets Mitochondria during Guanosine 5'-Triphosphate Guided Erythroid Differentiation. The International Journal of Biochemistry \& Cell Biology, 44, 1718-1728. http://dx.doi.org/10.1016/j.biocel.2012.06.023

[18] Hagenbuchner, J. and Ausserlechner, M.J. (2013) Mitochondria and FOXO3: Breath or Die. Frontiers in Physiology, 4, 147. http://dx.doi.org/10.3389/fphys.2013.00147

[19] Jacobs, K.M., Pennington, J.D., Bisht, K.S., Aykin-Burns, N., Kim, H.S., Mishra, M., Sun, L., Nguyen, P., Ahn, B.H., Leclerc, J., Deng, C.X., Spitz, D.R. and Gius, D. (2008) SIRT3 Interacts with the Daf-16 Homolog FoXO3a in the Mitochondria, as Well as Increases FOXO3a Dependent Gene Expression. International Journal of Biological Sciences, 4, 291-299. http://dx.doi.org/10.7150/ijbs.4.291

[20] Tang, B.L. (2006) SIRT1, Neuronal Cell Survival and the Insulin/IGF-1 Aging Paradox. Neurobiology of Aging, 27, 501-505. http://dx.doi.org/10.1016/j.neurobiolaging.2005.02.001

[21] Mouchiroud, L., Houtkooper, R.H., Moullan, N., Katsyuba, E., Ryu, D., Cantó, C., Mottis, A., Jo, Y.S., Viswanathan, M., Schoonjans, K., Guarente, L. and Auwerx, J. (2013) The NAD ${ }^{+} /$Sirtuin Pathway Modulates Longevity through Activation of Mitochondrial UPR and FoXO Signaling. Cell, 154, 430-441. http://dx.doi.org/10.1016/j.cell.2013.06.016

[22] Motta, M.C., Divecha, N., Lemieux, M., Kamel, C., Chen, D., Gu, W., Bultsma, Y., McBurney, M. and Guarente, L. (2004) Mammalian SIRT1 Represses Forkhead Transcription Factors. Cell, 116, 551-563. http://dx.doi.org/10.1016/S0092-8674(04)00126-6

[23] Olmos, Y., Sánchez-Gómez, F.J., Wild, B., García-Quintans, N., Cabezudo, S., Lamas, S. and Monsalve, M. (2013) SirT1 Regulation of Antioxidant Genes Is Dependent on the Formation of a FoxO3a/PGC-1 $\alpha$ Complex. Antioxidants \& Redox Signaling, 19, 1507-1521. http://dx.doi.org/10.1089/ars.2012.4713

[24] Aquilano, K., Vigilanza, P., Baldelli, S., Pagliei, B., Rotilio, G. and Ciriolo, M.R. (2010) Peroxisome Proliferator-Activated Receptor Gamma Co-Activator $1 \alpha(\mathrm{PGC}-1 \alpha)$ and Sirtuin 1 (SIRT1) Reside in Mitochondria: Possible Direct Function in Mitochondrial Biogenesis. Journal of Biological Chemistry, 285, 21590-21599. http://dx.doi.org/10.1074/jbc.M109.070169

[25] Yogev, O., Naamati, A. and Pines, O. (2011) Fumarase: A Paradigm of Dual Targeting and Dual Localized Functions. FEBS Journal, 278, 4230-4242. http://dx.doi.org/10.1111/j.1742-4658.2011.08359.x

[26] Yogev, O., Yogev, O., Singer, E., Shaulian, E., Goldberg, M., Fox, T.D. and Pines, O. (2010) Fumarase: A Mitochondrial Metabolic Enzyme and a Cytosolic/Nuclear Component of the DNA Damage Response. PLoS Biology, 8, e1000328. http://dx.doi.org/10.1371/journal.pbio.1000328

[27] Tomlinson, I.P.M., Rowan, A.J., Barclay, E., et al. (2002) Germline Mutations in FH Predispose to Dominantly Inherited Uterine Fibroids, Skin Leiomyomata and Papillary Renal Cell Cancer. Nature Genetics, 30, 406-410. http://dx.doi.org/10.1038/ng849

[28] Isaacs, J.S., Jung, Y.J., Mole, D.R., Lee, S., Torres-Cabala, C., Chung, Y.L., Merino, M., Trepel, J., Zbar, B., Toro, J., Ratcliffe, P.J., Linehan, W.M. and Neckers, L. (2005) HIF Overexpression Correlates with Biallelic Loss of Fumarate Hydratase in Renal Cancer: Novel Role of Fumarate in Regulation of HIF Stability. Cancer Cell, 8, 143-153. http://dx.doi.org/10.1016/j.ccr.2005.06.017

[29] Patel, M.S. and Korotchkina, L.G. (2006) Regulation of the Pyruvate Dehydrogenase Complex. Biochemical Society Transactions, 34, 217-222. http://dx.doi.org/10.1042/BST20060217

[30] Tang, B. (2014) The Mitochondrial Pyruvate Carrier and Metabolic Regulation. Cell Biology, 3, 111-117. http://dx.doi.org/10.4236/cellbio.2014.34011

[31] Zhou, Z.H., Liao, W., Cheng, R.H., Lawson, J.E., McCarthy, D.B., Reed, L.J. and Stoops, J.K. (2001) Direct Evidence for the Size and Conformational Variability of the Pyruvate Dehydrogenase Complex Revealed by Three-Dimensional Electron Microscopy. The "Breathing” Core and Its Functional Relationship to Protein Dynamics. The Journal of Bio- 
logical Chemistry, 276, 21704-21713. http://dx.doi.org/10.1074/jbc.M101765200

[32] Sutendra, G., Kinnaird, A., Dromparis, P., Paulin, R., Stenson, T.H., Haromy, A., Hashimoto, K., Zhang, N., Flaim, E. and Michelakis, E.D. (2014) A nuclear Pyruvate Dehydrogenase Complex Is Important for the Generation of Acetyl-CoA and Histone Acetylation. Cell, 158, 84-97. http://dx.doi.org/10.1016/j.cell.2014.04.046

[33] Chueh, F.Y., Leong, K.F., Cronk, R.J., Venkitachalam, S., Pabich, S. and Yu, C.L. (2011) Nuclear Localization of Pyruvate Dehydrogenase Complex-E2 (PDC-E2), a Mitochondrial Enzyme, and Its Role in Signal Transducer and Activator of Transcription 5 (STAT5)-Dependent Gene Transcription. Cellular Signalling, 23, 1170-1178. http://dx.doi.org/10.1016/j.cellsig.2011.03.004

[34] Hitosugi, T., Fan, J., Chung, T.W., Lythgoe, K., Wang, X., Xie, J., Ge, Q., Gu, T.L., Polakiewicz, R.D., Roesel, J.L., Chen, G.Z., Boggon, T.J., Lonial, S., Fu, H., Khuri, F.R., Kang, S. and Chen, J. (2011) Tyrosine Phosphorylation of Mitochondrial Pyruvate Dehydrogenase Kinase 1 Is Important for Cancer Metabolism. Molecular Cell, 44, 864-877. http://dx.doi.org/10.1016/j.molcel.2011.10.015

[35] Wellen, K.E., Hatzivassiliou, G., Sachdeva, U.M., Bui, T.V., Cross, J.R. and Thompson, C.B. (2009) ATP-Citrate Lyase Links Cellular Metabolism to Histone Acetylation. Science, 324, 1076-1080. http://dx.doi.org/10.1126/science.1164097

[36] Rea, S. (2001) CLK-1/Coq7p Is a DMQ Mono-Oxygenase and a New Member of the Di-Iron Carboxylate Protein Family. FEBS Letters, 509, 389-394. http://dx.doi.org/10.1016/S0014-5793(01)03099-X

[37] Vajo, Z., King, L.M., Jonassen, T., Wilkin, D.J., Ho, N., Munnich, A., Clarke, C.F. and Francomano, C.A. (1999) Conservation of the Caenorhabditis elegans Timing Gene CLK-1 from Yeast to Human: A Gene Required for Ubiquinone Biosynthesis with potential Implications for Aging. Mammalian Genome, 10, 1000-1004. http://dx.doi.org/10.1007/s003359901147

[38] Lakowski, B. and Hekimi, S. (1996) Determination of Life-Span in Caenorhabditis elegans by Four Clock Genes. Science, 272, 1010-1013. http://dx.doi.org/10.1126/science.272.5264.1010

[39] Takahashi, K., Noda, Y., Ohsawa, I., Shirasawa, T. and Takahashi, M. (2014) Extended Lifespan, Reduced Body Size and Leg Skeletal Muscle Mass, and Decreased Mitochondrial Function in CLK-1 Transgenic Mice. Experimental Gerontology, 58, 146-153. http://dx.doi.org/10.1016/j.exger.2014.08.003

[40] Monaghan, R.M., Barnes, R.G., Fisher, K., Andreou, T., Rooney, N., Poulin, G.B. and Whitmarsh, A.J. (2015) A Nuclear Role for the Respiratory Enzyme CLK-1 in Regulating Mitochondrial Stress Responses and Longevity. Nature Cell Biology, 17, 782-792. http://dx.doi.org/10.1038/ncb3170

[41] Jovaisaite, V., Mouchiroud, L. and Auwerx, J. (2014) The Mitochondrial Unfolded Protein Response, a Conserved Stress Response Pathway with Implications in Health and Disease. The Journal of Experimental Biology, 217, 137-143. http://dx.doi.org/10.1242/jeb.090738

[42] Schulz, A.M. and Haynes, C.M. (2015) UPR ${ }^{\mathrm{mt}}$-Mediated Cytoprotection and Organismal Aging. Biochimica et Biophysica Acta (BBA)-Bioenergetics, in press. http://dx.doi.org/10.1016/j.bbabio.2015.03.008

[43] Ng, F. and Tang, B.L. (2014) Pyruvate Dehydrogenase Complex (PDC) Export from the Mitochondrial Matrix. Molecular Membrane Biology, 31, 207-210. http://dx.doi.org/10.3109/09687688.2014.987183

[44] Nargund, A.M., Pellegrino, M.W., Fiorese, C.J., Baker, B.M. and Haynes, C.M. (2012) Mitochondrial Import Efficiency of ATFS-1 Regulates Mitochondrial UPR Activation. Science, 337, 587-590. http://dx.doi.org/10.1126/science.1223560

[45] Nargund, A.M., Fiorese, C.J., Pellegrino, M.W., Deng, P. and Haynes, C.M. (2015) Mitochondrial and Nuclear Accumulation of the Transcription Factor ATFS-1 Promotes OXPHOS Recovery during the UPR ${ }^{\mathrm{mt}}$. Molecular Cell, 58, 123-133. http://dx.doi.org/10.1016/j.molcel.2015.02.008

[46] Naamati, A., Regev-Rudzki, N., Galperin, S., Lill, R. and Pines, O. (2009) Dual Targeting of Nfs1 and Discovery of Its Novel Processing Enzyme, Icp55. The Journal of Biological Chemistry, 284, 30200-30208. http://dx.doi.org/10.1074/jbc.M109.034694

[47] Marelja, Z., Mullick Chowdhury, M., Dosche, C., Hille, C., Baumann, O., Löhmannsröben, H.G. and Leimkühler, S. (2013) The L-Cysteine Desulfurase NFS1 Is Localized in the Cytosol Where It Provides the Sulfur for Molybdenum Cofactor Biosynthesis in Humans. PLoS ONE, 8, e60869. http://dx.doi.org/10.1371/journal.pone.0060869

[48] Nakai, Y., Nakai, M., Hayashi, H. and Kagamiyama, H. (2001) Nuclear Localization of Yeast Nfs1p Is Required for Cell Survival. The Journal of Biological Chemistry, 276, 8314-8320. http://dx.doi.org/10.1074/jbc.M007878200 